JOSEF SCHILLER

AUSWAHL AUS SEINEM WERK 
TEXTAUSGABEN

ZUR FRUHHEN SOZIALISTISCHEN LITERATUR

IN DEUTSCHLAND

Begründet von BRUNO KAISER

und weitergeführt von URSULA MÚNCHOW

Herausgegeben vom

Zentralinstitut für Literaturgeschichte

der Akademie der Wissenschaften der DDR

BAND XXIII 


\section{JOSEF SCHILLER}

\section{AUSWAHL \\ AUS SEINEM WERK}

\section{Herausgegeben von}

NORBERT ROTHE

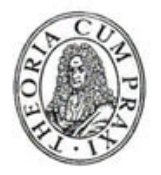

AKADEMIE-VERLAG · BERLIN 1982 
Erschienen im Akademie-Verlag

DDR - 1086 Berlin, Leipziger Straße 3-4

Lektor: Jutta Kolesnyk

(c) Akademie-Verlag Berlin 1982

Lizenznummer: $202 \cdot 100 / 172 / 82$

Gesamtherstellung: IV/2/14 VEB Druckerei

"Gottfried Wilhelm Leibniz", $445^{\circ}$ Gräfenhainichen $\cdot 5790$

Bestellnummer: 7539134 (2119/XXIII) - LSV 7105

Printed in GDR

DDR 16,- M 